\title{
BMJ Global Health Adolescent sexual and reproductive health research in sub-Saharan Africa: a scoping review of substantive focus, research volume, geographic distribution and Africa-led inquiry
}

To cite: Ajayi Al, Otukpa E0, Mwoka M, et al. Adolescent sexual and reproductive health research in subSaharan Africa: a scoping review of substantive focus, research volume, geographic distribution and Africa-led inquiry. BMJ Global Health 2021;6:e004129. doi:10.1136/ bmjgh-2020-004129

Handling editor Seye Abimbola

- Additional material is published online only. To view please visit the journal online (http://dx.doi.org/10.1136/ bmjgh-2020-004129).

Received 8 0ctober 2020 Revised 18 January 2021 Accepted 20 January 2021

Check for updates

(C) Author(s) (or their employer(s)) 2021. Re-use permitted under CC BY-NC. No commercial re-use. See rights and permissions. Published by BMJ.

Population Dynamics and Sexual and Reproductive Health and Rights Unit, African Population and Health Research Center, Nairobi, Kenya

Correspondence to Emmanuel Oloche 0tukpa; otukpa.emmanuel@gmail.com

\section{ABSTRACT}

Background Previous review studies have not systematically mapped the existing body of knowledge on adolescent sexual and reproductive health (ASRH) in sub-Saharan Africa (SSA). Our scoping review addresses this gap by examining how the body of research on ASRH in SSA has evolved over the past decade, and its present profile, in terms of trends in volume, geographic and substantive focus, and Africa-led inquiry. Methods We used a three-step search strategy to identify English and French peer-reviewed publications and relevant grey literature on ASRH in SSA published between January 2010 and December 2019. Two reviewers screened the titles, abstracts and full texts of publications for eligibility and inclusion.

Results A total of 1302 articles were published over the period, rising from 91 in 2010 to 183 in 2015. However, the bulk of the studies (63.9\%) focused on six (South Africa, Kenya, Nigeria, Tanzania, Uganda and Ethiopia) of the 46 SSA countries. Ten countries had no ASRH papers, while five others each had only one publication. While issues like HIV (17.2\%), sexual behaviours (17.4\%) and access to sexual and reproductive health services $(13.0 \%$ ) received substantial attention, only a few studies focused on early adolescence (1014 years), programme interventions, scaling up of interventions and policy evaluation. Just over half of publications had authors with African institutional affiliations as first authors (51.1\%) or last author (53.0\%). Sixteen per cent of papers did not include any authors from institutions in Africa.

Conclusions Our review demonstrated that research on ASRH is limited in focus and is unevenly distributed across SSA countries. The identified gaps can guide future research and funding to advance ASRH policies and programmes. It is also vital for stakeholders in the research enterprise, including researchers, donors, ethical review boards, and journal editors and reviewers, to implement measures that foster national investigators' inclusion.

\section{INTRODUCTION}

Adolescents (aged 10-19 years) in subSaharan Africa (SSA) bear a huge and disproportionate burden of poor sexual and

\section{Key questions}

What is already known?

- There is a large body of studies on adolescent sexual and reproductive health (ASRH) outcomes in sub-Saharan Africa (SSA). However, no study has systematically mapped the existing body of knowledge, both in terms of substantive and geographic focus in order to inform further evidence generation.

What are the new findings?

- Our analysis reveals a rise in ASRH research in SSA between 2010 and 2019. However, the bulk of research was conducted in six of the 46 SSA countries, with 10 countries having no publication.

- While topics like HIV, access to contraceptives and sexual behaviours have received significant attention, issues like sexually transmitted infections, comprehensive sexuality education and adolescent and youth-friendly services, menstrual hygiene and gender norms, as well as studies focusing on early adolescents, received little attention.

- Just over half of the articles are led by authors with affiliations to African institutions.

What do the new findings imply?

- Stakeholders in ASRH research including donors researchers and governments should prioritise research in understudied ASRH topics and countries.

- The low representation of local authors in ASRH research may reflect under-investment in research and research capacity building in many SSA countries and power differentials in knowledge creation. The limited number of locally led research outputs highlights the need for investments in research capacity strengthening to enhance local production of ASRH knowledge.

reproductive health (SRH) outcomes. ${ }^{1-5}$ The SSA region accounts for the highest burden of adolescent HIV globally, and adolescent girls, in particular, are disproportionately affected, 
accounting for three out of four new HIV infections. ${ }^{6}$ The prevalence of adolescent pregnancy, unsafe abortion, child marriage, sexual and gender-based violence (SGBV) and female genital mutilation (FGM) is also substantially higher in SSA compared with other regions of the world. ${ }^{7-9}$ Research has shown that pregnancyrelated complications and HIV/AIDS are the leading causes of death among adolescents in SSA. ${ }^{10}$

Morbidities and disabilities from unsafe abortion, birth complications, SGBV and FGM not only threaten adolescents' immediate physical and mental health but also have adverse long-term health ${ }^{811}$ and socioeconomic consequences. ${ }^{12} 13$ For instance, child marriage and early childbearing are known drivers of poverty and gender inequalities in economic and educational outcomes. ${ }^{14-16}$ Early and unintended pregnancy often disrupts girls' education, with most pregnant and parenting adolescents dropping out of school and rarely returning, ${ }^{17}$ with long-term impacts on skills building, livelihood opportunities and earning potential. Adolescent pregnancies also have adverse implications for child health. Babies born to adolescent mothers face a higher risk of being born preterm, underweight and of dying before their fifth birthday. ${ }^{18-20}$ Children of adolescent mothers are also more likely to have behavioural problems, have lower educational achievement and become adolescent parents themselves. ${ }^{21}$ These disadvantages, exacerbated by their parents' poor socioeconomic status, may become more pronounced as they grow older, making them susceptible to poor health and socioeconomic outcomes, and increasing the likelihood of a multigenerational cycle of increased risk for adverse outcomes.

The barriers that heighten adolescents' vulnerability to poor SRH outcomes in SSA are complex and multifaceted. At the individual level, barriers include a lack of knowledge of and negative attitudes to contraceptives. ${ }^{22-24}$ At the family level, lack of parental support, care and attention can lead to poor SRH outcomes. ${ }^{25}$ Unequal gender norms, harmful traditional practices, poverty, violence, exclusion from SRH services and information, and lack of progressive SRH policies are some of the structural factors that heighten adolescents' risk for poor SRH outcomes. ${ }^{23} 26-28$

African countries have articulated several collective commitments to address these barriers and improve adolescents' health and well-being. Examples of such frameworks are the Protocol to the African Charter on Human and Peoples' Rights on the Rights of Women in Africa (the Maputo Protocol), Addis Ababa Declaration on Population and Development, Agenda 2063-the Africa we want, African Youth Charter, African Union Campaign to End Child Marriage and African Charter on the Rights and Welfare of the Child. ${ }^{29-35}$ Even though these commitments hold promise in redressing adolescents' poor SRH outcomes, progress at domestication and implementation through progressive legislation, policies and guidelines at national levels remain slow. ${ }^{30} 31$ Obstacles to progress are, in part, the absence of required evidence, synthesised and appropriately channelled to inform national-level policy debates. ${ }^{30}$ Understanding the landscape of and gaps in existing research on adolescent sexual and reproductive health (ASRH) in SSA is needed to help address both barriers.

To meet this need, we conducted a comprehensive scoping review of ASRH research in SSA to identify key ASRH topics and countries for which a knowledge base exists, summarise this knowledge and pinpoint major areas, both substantive and geographical, where further evidence generation is required. In this paper, we examine how the body of research on ASRH in SSA has evolved over the past decade, and its present profile, in terms of trends in volume, geographic and substantive focus, research approaches and African-led inquiry. The renewed calls for decolonising global health made prominent by the under-representation of Africa in publications from data generation from Africa necessitates our focus on African-led inquiry in ASRH research. ${ }^{36}{ }^{37}$ Also, political leaders are responsible for developing strategies to address poor ASRH outcomes in the region. Political leaders can be suspicious about foreign-driven research (funded by foreign, international non-governmental organisations and conducted by foreign researchers) that is intended to inform local strategies to improve ASRH.

\section{METHODS \\ Protocol and registration}

We adopted the methodological framework proposed by Arksey and $\mathrm{O}^{\prime} \mathrm{Malley}^{38}$ in designing this study, and the Preferred Reporting Items for Systematic Reviews and Meta-analyses (PRISMA) extension for scoping reviews' checklist guided our presentation in this paper. ${ }^{39}$ Our study protocol was published in BMJ Open in July $2020{ }^{40}$

\section{Search strategy}

We searched for papers on ASRH in SSA published between January 2010 and December 2019. To ensure a comprehensive and exhaustive search, we first conducted a limited preliminary search of PubMed, Web of Science and JSTOR using the keywords 'adolescent sexual and reproductive health' AND 'sub-Saharan Africa'. We then analysed the index terms used in describing the articles from our preliminary search to prepare the comprehensive controlled vocabulary for our main search as detailed in the study protocol. ${ }^{40}$ We used the Peer Review of Electronic Search Strategies 2015 guideline checklist to assess, evaluate and revise our controlled vocabulary before conducting our main search. We performed the search using all identified Medical Subject Headings (MeSH) terms (see online supplemental appendix 1). To ensure that we included all relevant studies and particularly those published in African journals, we searched the following online bibliographic databases: PubMed, Web of Science, African Journals Online, JSTOR, HINARI, Scopus, PsycInfo, Science Direct and Google Scholar. We also searched Google and websites of UNICEF, WHO, 
UNFPA and the Guttmacher Institute to identify relevant grey literature. Finally, we searched the reference list of all review studies found in the search.

\section{Eligibility criteria}

We included only articles and reports published in English and French languages between January 2010 and December 2019. Our decision to include papers published in the last decade mirrors our unique need for recent evidence to support advocacy on the domestication and implementation of continental frameworks to enhance ASRH in SSA. The ASRH topics included are exhaustive and broad. ${ }^{41-44}$ The topics are included in the data extraction form (online supplemental appendix 2).

We included papers focusing on adolescents aged 10-19 years in SSA. Studies that primarily focused on women of reproductive age (15-49 years) or youths aged 15-35 years were excluded if they did not expressly provide age disaggregated data specific to adolescents. Studies that reported on 20-24year-olds were included only if they retrospectively reported data from when these participants were aged 10-19 years old or if they focused on topics like child marriage, FGM and age at sexual debut. All studies, irrespective of the research methods used-qualitative, quantitative, mixed-methods studies and reviews-were included. We included peer-review publications, theses and technical reports on primary research. However, we excluded theses and technical reports based on data that had also been published in peer-review journals to avoid double counting. We also excluded conference proceedings, commentaries, abstracts, book reviews, blog posts and other kinds of grey literature.

\section{Study selection}

Two reviewers independently screened titles, abstracts and full texts using Covidence. ${ }^{45}$ We included articles that both reviewers agreed should be included using the pre-specified inclusion criteria. In cases of unresolved disagreement, another member of the research team provided a third opinion. Figure 1 presents the PRISMA flow diagram to demonstrate the process of article screening, inclusion and exclusion. The initial search conducted by the researchers yielded 12735 titles, from which we removed 3469 duplicates. We screened the titles and abstracts of the remaining 8723 studies and excluded 7152 studies that did not meet the inclusion criteria. We assessed a total of 1570 full-text papers and further excluded 268 that did not meet our inclusion criteria, leaving a total of 1302 studies in our analysis.
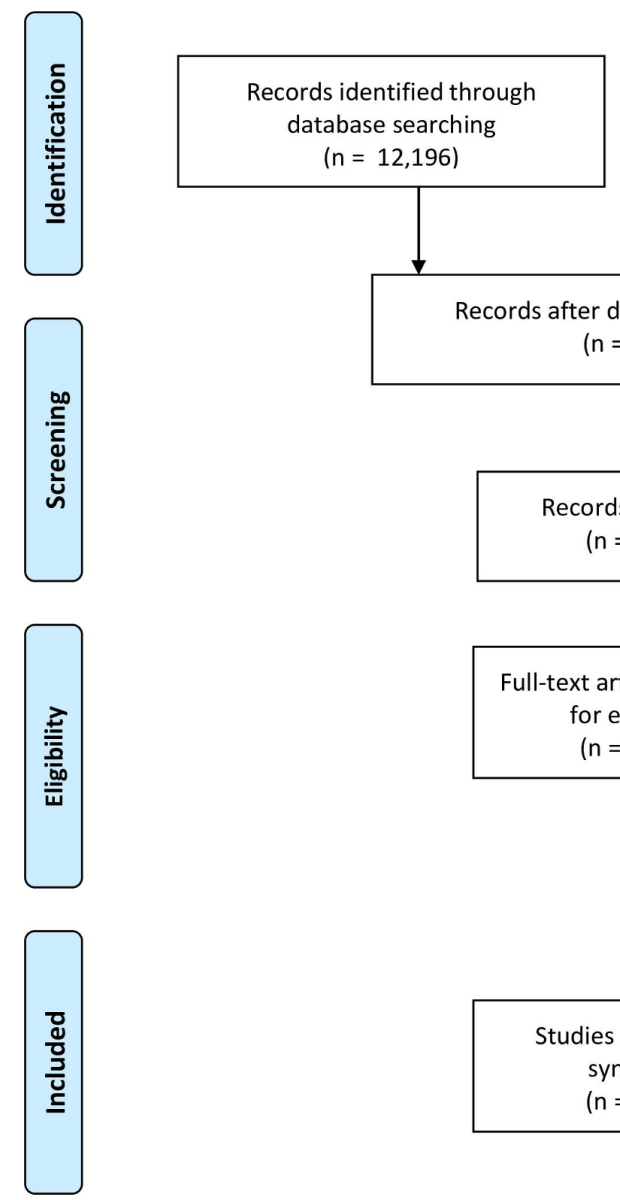

Additional records identified through other sources $(n=539)$
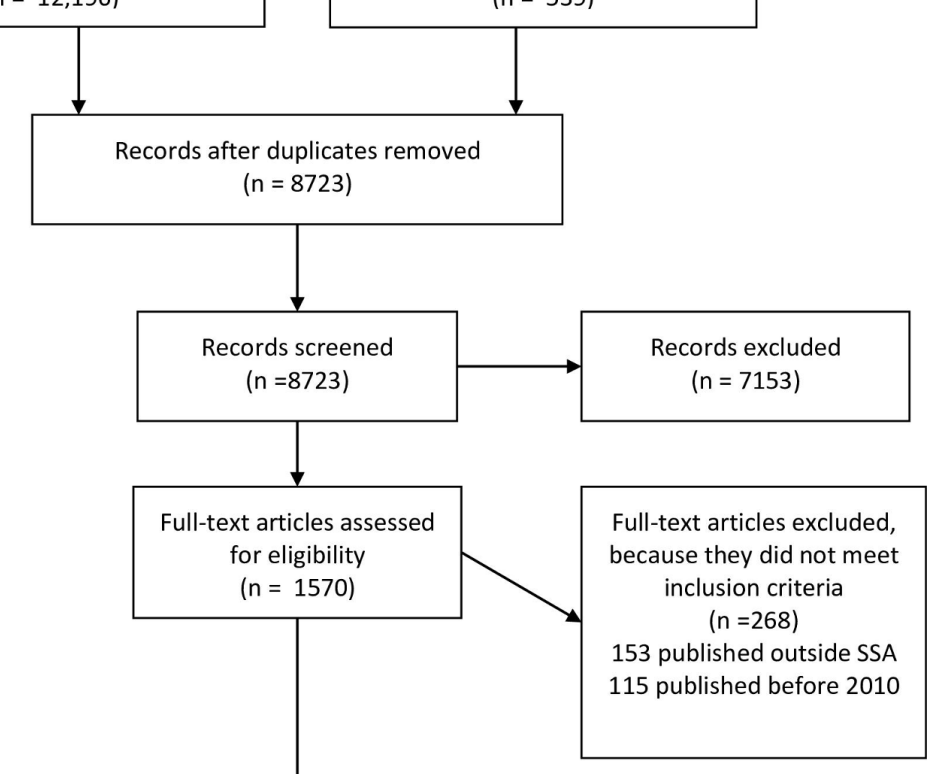

Studies included in synthesis

( $n=1302)$

Figure 1 Overview of the article selection (PRISMA flow diagram). PRISMA, Preferred Reporting Items for Systematic Reviews and Meta-analyses; SSA, sub-Saharan Africa. 


\section{Data extraction and charting}

We extracted data from included articles using a standardised form in Microsoft Excel. Specifically, we extracted the country of the first and last authors using their affiliations. We classified papers as African-led if the author was affiliated with an institution in the country of study or another African country. We also extracted information on collaboration type by classifying the article into seven different geographic collaboration types based on a previous study ${ }^{46}$ : (1) papers authored by individuals from the country of focus only, (2) papers whose authors included individuals from US top 20 universities based on Webometrics' ranking, (3) papers whose authors included individuals from the USA and Canada but not top US universities, (4) authors included individuals from Europe, (5) authors included individuals from other African countries, (6) authors included individuals from multiple locales, (7) authors included individuals from Asian and Australia. Also, we extracted information on journal coverage and impact factors. Journal coverage was classified as (a) African or regional journal, (b) international journal or (c) national journal (in-country). We determined the coverage of a journal by reading the 'About section' on the journal's webpage and classified them as international, regional and national. To classify articles under a substantive focus, we first conducted a quick review of previous review articles to determine common ASRH themes in the literature. ${ }^{9} 4347$ We then classified all articles under these themes, creating new themes for those not appropriately fitting under existing categories. Other information extracted from articles included in the study is presented in online supplemental appendix 2. Five research assistants were trained to conduct the data extraction using the standardised Microsoft Excel form. The research team reviewed samples of the extracted data for quality assurance.

\section{Patient and public involvement}

Patients and the public were not involved in the preparation of this study.

\section{Analysis}

We used descriptive statistics to summarise the volume of studies, trends, authorship, country of publication and substantive focus. We cross-tabulated the papers by country and region of research to determine the gaps in ASRH publication in geography. All analyses were conducted in R-Studio V.1.2.5033, and Microsoft Excel 2019.

\section{RESULTS}

\section{Volume of evidence}

Figure 2 presents the trends in the number of publications over the review period. The results were normally distributed, peaking in $2015 \quad(\mathrm{n}=183)$, and dipping significantly after. Table 1 describes the characteristics of the 1302 studies. A subregional overview shows that East Africa produced the highest number of ASRH

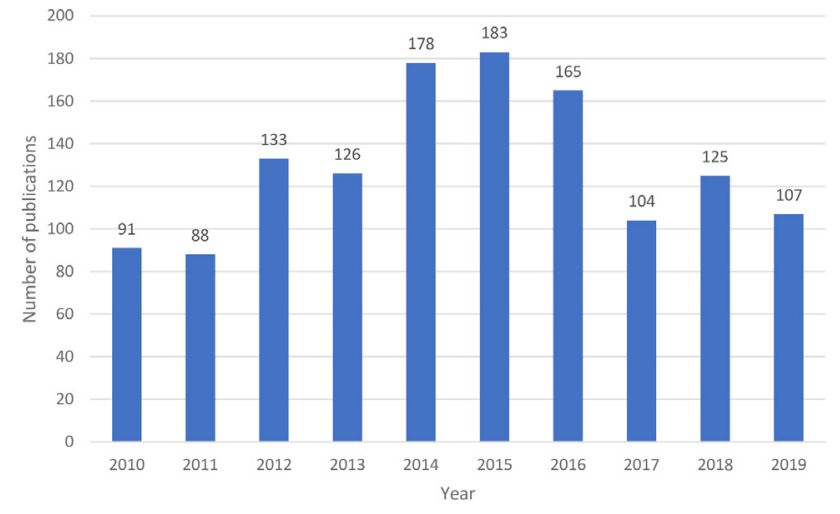

Figure 2 Trends in ASRH publication in SSA: number of studies between 2010 and 2019. ASRH, adolescent sexual and reproductive health; SSA, sub-Saharan Africa.

studies (37.6\%), followed by Southern Africa (28.7\%). The majority of ASRH studies had multiple authors $(81.6 \%)$, and most were published in international journals $(78.1 \%)$ and journals with an impact factor of 1-4 $(61.2 \%)$. Only a few studies were published in journals with impact factors above $4(6.4 \%)$. While over half of the ASRH studies adopted purely quantitative methods $(52.7 \%)$, only $23.7 \%$ used purely qualitative methods.

\section{Substantive focus}

As shown in table 2, the substantive focus of ASRH studies was mainly on HIV (17.2\%), sexual behaviours (17.4\%), access to SRH services (13.0\%), SGBV (6.3\%), adolescent pregnancy $(5.8 \%)$ and child marriage $(5.1 \%)$.

There was a huge between-country disparity in the number of ASRH publications focusing on HIV in SSA. Approximately $66 \%$ of studies focusing on HIV were conducted in Eastern and Southern African countries. However, South Africa alone accounted for about a quarter of all HIV-related publications. The HIV thematic focus mainly included HIV prevalence (28\%), HIV risk $(15.1 \%)$, challenges faced by adolescents living with HIV (13.8\%), HIV risk reduction interventions (12.9\%), HIV prevention (12.1\%), adherence to antiretroviral therapy (ART) (7.3\%), HIV testing (6.9\%), ART initiation $(6.0 \%)$, status disclosure $(5.6 \%)$ and HIV knowledge (5.2\%). The number of ASRH publications focusing on HIV increased between $2010(\mathrm{n}=16)$ and $2016(\mathrm{n}=35)$ but declined from $2017(\mathrm{n}=17)$ to $2019(\mathrm{n}=14)$.

Access to SRH services was the second most common area of focus. East (38.2\%) and Southern Africa (26.4\%) accounted for most of the papers included on adolescent access to SRH services. Most papers on adolescent access to SRH services focused on South Africa (18.0\%), Tanzania $(10.7 \%)$, Kenya $(10.1 \%)$, Ethiopia $(8.4 \%)$, Uganda $(7.9 \%)$, Nigeria $(6.7 \%)$ and Ghana $(5.1 \%)$. There was no clear pattern in trends in the volume of publications on access to SRH services.

Approximately $43 \%$ of publications focusing on sexual behaviours focused on East African countries. However, South Africa (17.7\%), Nigeria (13.7\%), Kenya (12.4\%), 


\begin{tabular}{|c|c|c|}
\hline Paper characteristics & $\begin{array}{l}\text { Frequency } \\
n=1302\end{array}$ & Per cent \\
\hline \multicolumn{3}{|l|}{ Region of paper's focus } \\
\hline East Africa & 489 & 37.6 \\
\hline Southern Africa & 375 & 28.7 \\
\hline West/central Africa & 242 & 18.6 \\
\hline Sub-Saharan Africa & 146 & 11.3 \\
\hline $\begin{array}{l}\text { Global Low and Middle } \\
\text { Income Countries (LMIC) }\end{array}$ & 50 & 3.8 \\
\hline \multicolumn{3}{|l|}{$\begin{array}{l}\text { Number of authors on the } \\
\text { paper }\end{array}$} \\
\hline Multiple authors & 1066 & 81.9 \\
\hline Single author & 236 & 18.1 \\
\hline \multicolumn{3}{|l|}{ Journal coverage } \\
\hline International journal & 1017 & 78.1 \\
\hline African/regional & 149 & 11.4 \\
\hline National & 136 & 10.4 \\
\hline \multicolumn{3}{|l|}{ Impact factor } \\
\hline No impact factor & 220 & 16.9 \\
\hline$<1$ & 202 & 15.5 \\
\hline $1-2.5$ & 480 & 36.9 \\
\hline $2.6-4$ & 316 & 24.3 \\
\hline 4.1 and above & 84 & 6.4 \\
\hline \multicolumn{3}{|l|}{ Research methods } \\
\hline Quantitative & 685 & 52.7 \\
\hline Cross-sectional & 578 & 44.4 \\
\hline $\begin{array}{l}\text { Randomised controlled } \\
\text { trial }\end{array}$ & 60 & 4.6 \\
\hline Quasi-experimental & 15 & 1.2 \\
\hline Pilot study & 8 & 0.6 \\
\hline Cohort & 17 & 1.3 \\
\hline Other designs & 7 & 0.5 \\
\hline Qualitative & 309 & 23.7 \\
\hline Mixed methods & 164 & 12.6 \\
\hline Review & 141 & 10.8 \\
\hline Neither & 3 & 0.2 \\
\hline \multicolumn{3}{|l|}{ Age group of research focus } \\
\hline $10-14$ & 116 & 8.9 \\
\hline $10-19$ & 875 & 67.1 \\
\hline $15-19$ & 312 & 24.0 \\
\hline \multicolumn{3}{|l|}{ Paper's collaborative type } \\
\hline $\begin{array}{l}\text { Only authors from the } \\
\text { country of focus }\end{array}$ & 453 & 34.8 \\
\hline $\begin{array}{l}\text { Authors include individuals } \\
\text { from US top universities }\end{array}$ & 102 & 7.8 \\
\hline
\end{tabular}

Continued
Table 1 Continued

\begin{tabular}{|lcc|}
\hline Paper characteristics & $\begin{array}{l}\text { Frequency } \\
\mathrm{n}=\mathbf{1 3 0 2}\end{array}$ & Per cent \\
\hline $\begin{array}{l}\text { Authors include individuals } \\
\text { from USA/Canada but not } \\
\text { top US university (only) }\end{array}$ & 270 & 20.7 \\
$\begin{array}{l}\text { Authors include individuals } \\
\text { from Europe }\end{array}$ & 260 & 20.0 \\
$\begin{array}{l}\text { Authors include individuals } \\
\text { from other Africa countries }\end{array}$ & 46 & 3.5 \\
$\begin{array}{l}\text { Authors include individuals } \\
\text { from multiple (more than } \\
\text { three places) locales }\end{array}$ & 152 & 11.7 \\
$\begin{array}{l}\text { Authors include individuals } \\
\text { from Australia or Asia }\end{array}$ & 19 & \\
\hline
\end{tabular}

Paper includes author from the 1006

77.3 country of focus

Papers with first authors
with an African-institutional

Papers with middle authors $\quad 291 \quad 22.4$
with an African-institutional 2.4 affiliation

Papers with last authors
with an African-institutional

Papers that do not include $\quad 204 \quad 15.7$
an author with an Africaninstitutional affiliation

\begin{tabular}{lrr} 
Country of funders & & \\
USA & 273 & 21.1 \\
UK & 94 & 7.2 \\
Canada & 17 & 1.3 \\
\hline EU countries & 103 & 7.9 \\
\hline South Africa & 30 & 2.3 \\
Ethiopia & 23 & 1.7 \\
Other African countries & 25 & 1.9 \\
\hline Other locales (Australia, & 8 & 0.6 \\
Japan and Taiwan) & & 55.9 \\
\hline Not funded & 728 & 1.9 \\
\hline
\end{tabular}

$\mathrm{ASRH}$, adolescent sexual and reproductive health; LMIC, low and middle income country.

Uganda $(11.5 \%)$, Tanzania $(9.3 \%)$ and Ethiopia $(9.3 \%)$ had the most publications on adolescent sexual behaviours. There was no clear pattern in changes in the number of publications from year to year.

Of the 82 papers published on SGBV among adolescents, a quarter $(23.2 \%)$ reported on studies conducted in South Africa. We did not find any SGBV papers in other Southern African countries. Publications on SGBV among adolescents slightly increased between $2010(n=1)$ and $2017(\mathrm{n}=12)$. 
Table 2 ASRH papers stratified by substantive focus

\begin{tabular}{|c|c|c|}
\hline Substantive focus & $\begin{array}{l}\text { Frequency } \\
\text { counts }\end{array}$ & Per cent \\
\hline HIV (all aspects) & 231 & 17.7 \\
\hline $\begin{array}{l}\text { Sexual behaviour and } \\
\text { transactional sex }\end{array}$ & 226 & 17.4 \\
\hline Access to SRH services & 223 & 17.9 \\
\hline $\begin{array}{l}\text { Sexual and gender-based } \\
\text { violence }\end{array}$ & 82 & 6.3 \\
\hline Adolescent pregnancy & 76 & 5.8 \\
\hline Child marriage & 67 & 5.1 \\
\hline Female genital mutilation & 62 & 4.8 \\
\hline School-based interventions & 60 & 4.6 \\
\hline Sex initiation & 54 & 4.1 \\
\hline $\begin{array}{l}\text { Comprehensive sexuality } \\
\text { education }\end{array}$ & 47 & 3.6 \\
\hline Menstrual hygiene & 46 & 3.5 \\
\hline Male circumcision & 44 & 3.4 \\
\hline Human papilloma virus & 43 & 3.3 \\
\hline Drug use/mental health & 41 & 3.1 \\
\hline Social/gender norm & 34 & 2.6 \\
\hline $\begin{array}{l}\text { Adolescent youth-friendly } \\
\text { services }\end{array}$ & 26 & 2.0 \\
\hline STI prevalence & 25 & 1.9 \\
\hline $\begin{array}{l}\text { Large scale campaigns, } \\
\text { community mobilisation, peer- } \\
\text { focused interventions and } \\
\text { engagement of boys }\end{array}$ & 25 & 1.9 \\
\hline Economic empowerment of girls & 22 & 1.7 \\
\hline Laws and policies & 18 & 1.4 \\
\hline Parental engagement & 18 & 1.4 \\
\hline
\end{tabular}

$\mathrm{ASRH}$, adolescent sexual and reproductive health; $\mathrm{SRH}$, sexual and reproductive health; STI, sexually transmitted infection.

Most of the papers on adolescent pregnancy reported on Southern Africa (38.1\%) and West/Central Africa $(23.7 \%)$. However, South Africa (25\%), Ghana (13.2\%) and Ethiopia $(9.2 \%)$ had the most publications on adolescent pregnancy. There was no clear pattern in terms of trends.

Two-fifths of publications on child marriage focused on East Africa, with Ethiopia having the most papers $(19.4 \%)$, followed by Kenya $(10.5 \%)$ and Tanzanian $(6.0 \%)$. Papers on child marriage increased from one in 2010 and five in 2011 to 13 in 2015 and 10 in 2019.

\section{Geographic distribution of evidence}

Figure 3 shows the geographic distribution of ASRH papers. The bulk of studies $(63.9 \%)$ published in the past decade focused on six countries (South Africa, 19.2\%; Kenya, 12.1\%; Nigeria, 9.1\%; Tanzania, 8.0\%; Uganda, $8.0 \%$ and Ethiopia, $7.5 \%$ ). In the West and Central African subregions, $74.8 \%$ of papers focused

$$
\text { [-10 } 11-20 \square 21-30 \square 41-100=>100
$$

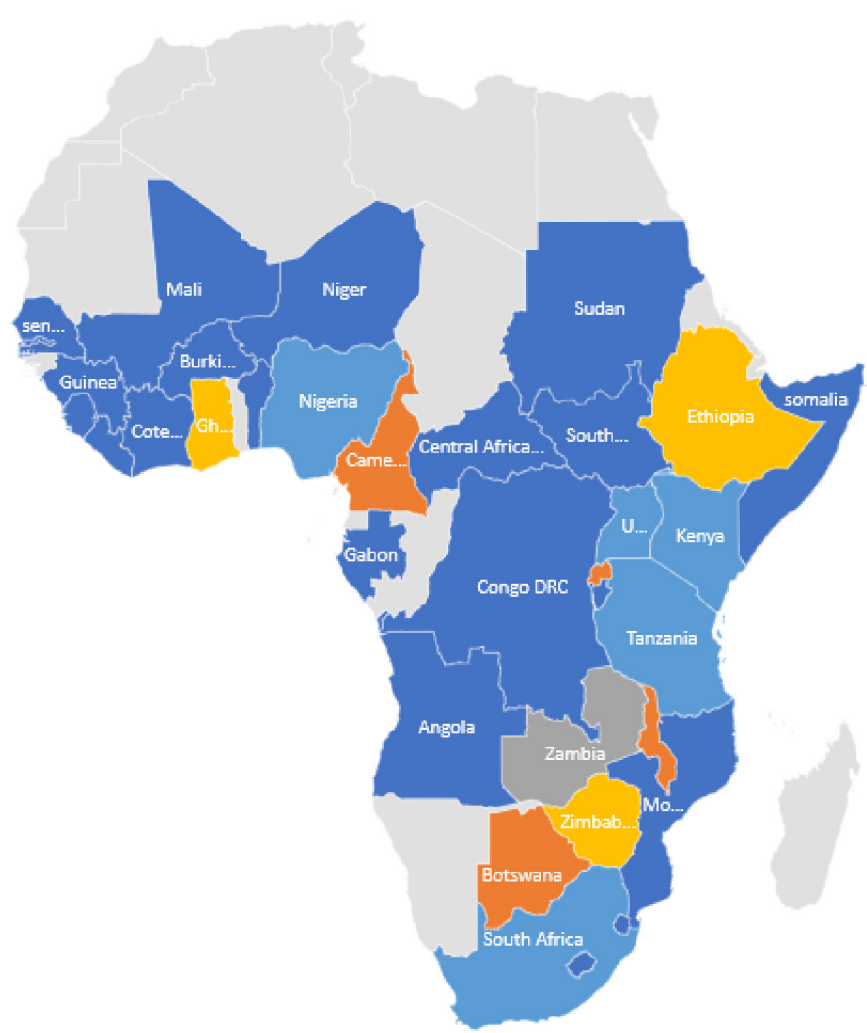

Figure 3 Geographic distribution of SSA ASRH papers. ASRH, adolescent sexual and reproductive health; SSA, subSaharan Africa.

on Ghana and Nigeria. In East Africa, Kenya (32.1\%), Tanzania (21.3\%), Uganda $(21.3 \%)$ and Ethiopia $(19.4 \%)$ (online supplemental table 1) accounted for the highest number of ASRH papers. The majority (66.8\%) of papers on Southern Africa focused on South Africa. We found no ASRH paper focused on the following 10 countries: Madagascar, Namibia, Togo, Liberia, Guinea Bissau, Equatorial Guinea, Mauritius, Mauritania, Chad or Eritrea. Only one ASRH paper each was found in these countries: Niger, Benin Republic, Burundi, Cape Verde, Gabon and the Central Africa Republic.

\section{African-led ASRH papers and collaboration types}

Seventy-seven per cent of the papers included in the review had at least one coauthor from an institution based in the country of focus, and $86 \%$ had an author affiliated to an institution based in Africa. Slightly more than half of the papers had individuals affiliated to African institutions as first author $(51.1 \%)$ or last author $(53.0 \%)$. Papers focusing on nine countries (Benin, Burundi, Cape Verde, Gabon, Guinea, Ivory Coast, Niger, Senegal and Somalia) had no authors affiliated with an African institution (figure 4). In contrast, all papers based on data from Angola, Liberia and the Democratic Republic of Congo had at least one local author. Authorship by Africa-based scholars increased slightly between 2010 and 2016, as shown in figures 4 and 5. However, it declined in 2017 and rose in 2018. 


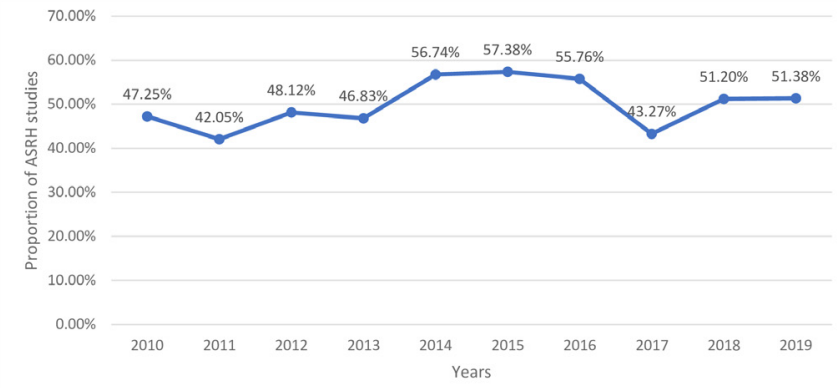

Figure 4 Trend in African affiliated first author ASRH research. $\mathrm{ASRH}$, adolescent sexual and reproductive health.

\section{Collaboration in ASRH research}

We analysed the collaboration types in ASRH research in SSA. As shown in table 1, 34.8\% of the ASRH studies had authors only from the country of the study's focus. The rest had collaborators, mostly from Europe and the USA. Close to half of the ASRH papers (48.5\%) had authors affiliated with institutions in Europe, the USA and Canada. Collaboration among Africa authors was low, with only $3.5 \%$ of ASRH studies having authors from different African countries only. When African authors collaborated with researchers from Europe and the USA, they were less likely to be the lead author, as shown in figure 6 .

\section{DISCUSSION}

We examined how the body of research on ASRH in SSA has evolved over the past decade, its present profile, in terms of trends in volume, geographic and substantive focus, research approaches and Africa-led inquiry. Our analysis reveals a rise in ASRH research in SSA between 2010 and 2019, peaking in 2015. Overall, the volume of research in SSA in the past decade is low, with only around 130 publications per year and many countries having one paper or none at all. The finding that ASRH research peaked around 2015 might reflect the increasing commitment and focus on ASRH at international and regional fora. ${ }^{48}$ More SRH programmes and research were implemented as part of the late push to realise or evaluate progress towards the Millennium Development Goal 5.

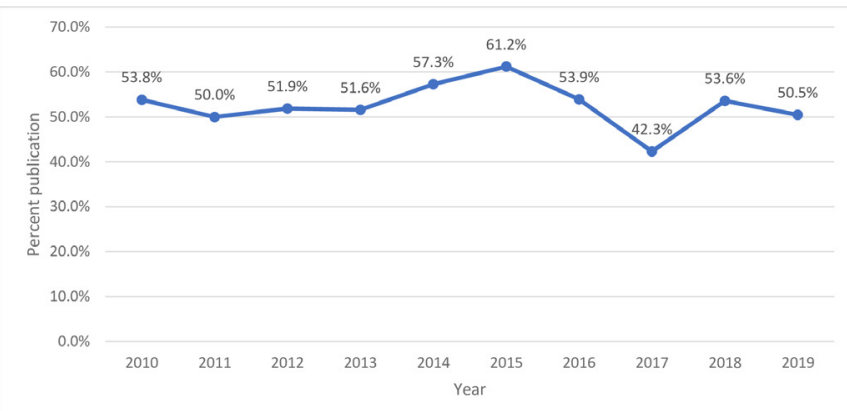

Figure 5 Trend in African affiliated last author ASRH research. $\mathrm{ASRH}$, adolescent sexual and reproductive health.

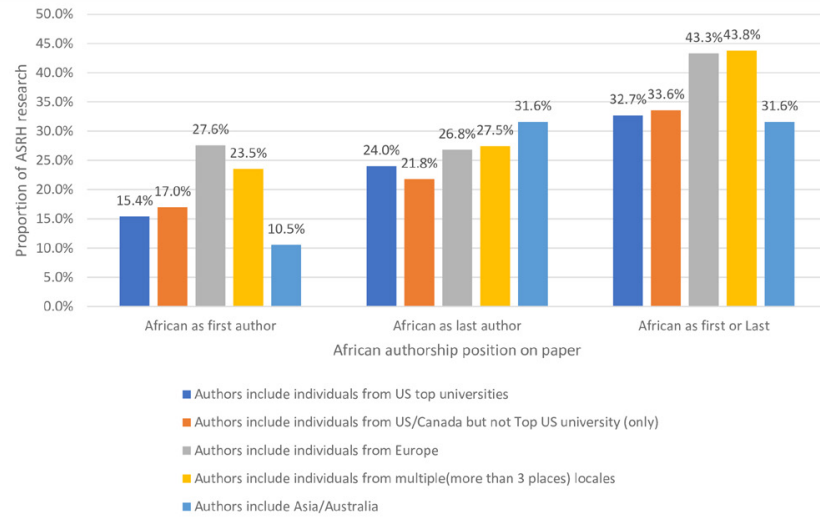

Figure 6 Research collaboration types stratified by African lead authorship. ASRH, adolescent sexual and reproductive health.

The significant focus on HIV, sexual behaviours and transactional sex likely stems from the huge burden of HIV in SSA, which has attracted significant research funds from global developmental partners working to reduce the rate of new infections and end AIDS-related deaths. Our findings underscore the need for research on other STIs, comprehensive sexuality education and adolescent and youth-friendly services, menstrual hygiene, and gender norms, early adolescence (the period when adolescents are aged 10-14 years), programme interventions, scaling up of interventions and policy evaluation. The latter research is particularly needed to assess existing policies and inform efforts to strengthen their implementation. Our findings also show that even for relatively wellstudied topics, gaps exist in terms of geographic coverage, with most SSA countries having limited research. As such, funders and researchers should prioritise topics and settings where limited research exists.

Our review's key finding is that the distribution of ASRH research was uneven, with most ASRH studies conducted in six of the $46 \mathrm{SSA}$ countries. Ten countries had no ASRH publication, and five had only one paper. This finding underscores the geographic gaps in ASRH research in SSA, suggesting a need for funders and researchers to prioritise studies in under-researched SSA settings. It is also critical to develop the research ecosystem in these countries to build ASRH research experts who will define key research priorities for their countries. ${ }^{49}$ This need is a priority given that national-level research is critical in informing policy debates and advocacy for progressive policies and guidelines. Politicians, leaders and policy actors need evidence to advance adolescents' health and well-being in many SSA countries lacking ASRH research. As such, there is a need to study the ASRH outcomes in under-researched countries. Programmes like the Consortium for Advanced Research Training in Africa (CARTA) have a demonstrable impact in developing the research ecosystem in SSA. ${ }^{50-53}$ Even though we did not examine the contributions of Masters and $\mathrm{PhD}$ students who benefited from research capacity building programmes being implemented on the continent to ASRH research, 
an evaluation of the CARTA programme has shown an increase in recipients' research outputs. ${ }^{54}$ The CARTA programme is an Africa-led initiative, with funding from global developmental partners (SIDA, Wellcome Trust and Carnegie Foundation among others), which seeks to rebuild and strengthen African universities' capacity to produce skilled researchers, capable of generating quality research to improve population health in Africa. However, such programmes often focus on and benefit SSA countries where a substantial knowledge base already exists, neglecting under-researched settings. There is, therefore, a need to consciously and intentionally prioritise research and research capacity building and mentoring programmes in SSA countries where the ASRH knowledge base is limited.

One in six articles did not include any local authors, and local researchers led a little over half of ASRH research. Further, when African authors collaborated with researchers from Europe and the USA, they were less likely to be the lead author. This observation was more frequent for papers with authors from the USA than Europe. For example, Africans were the first author in more than one-quarter of papers with European affiliated collaborators compared with only $17 \%$ of those with US-based authors. These findings are consistent with previous studies. ${ }^{465}$ Several factors could explain these results. The low representation of Africa-based authors in the lead author position may reflect their limited access to research resources as well as power imbalances in knowledge production. However, it is worth noting that although Africa-based authors may have substantially contributed to the research, the publication and research may simply not happen without the US-based or EU-based authors. This is because the primary funding for ASRH studies in SSA comes from the USA and Europe, and authors from these countries are more likely to receive these research funds. In most cases, they not only come with the funding but also have the expertise and design the research. So, it is not surprising that foreign authors led a substantial number of ASRH research in the region. However, given that under-representation of African scholars in lead authorship positions perpetuates power imbalances in knowledge production, it is imperative that funders, journals and research institutions develop transformative policies and principles that address the imbalances and ensure that local researchers are accorded more prominence.

Relatedly, governments' investment in research is generally low in most SSA countries, except for South Africa, resulting in limited funding for African researchers to conduct research. ${ }^{56}$ Most SSA countries also have inadequate research infrastructure and resources for training and mentoring, limiting the number of qualified researchers. ${ }^{56} 57$ A previous study has shown that Africa has only 198 researchers per million inhabitants compared with over 4000 in the global north. ${ }^{58}$ Fulfilment of the African Union's recommendation of expenditure of $1 \%$ of gross domestic product on research and development $t^{59}$ will go a long way in increasing African researchers' outputs and the number of qualified researchers. Building research infrastructure will significantly impact SSA countries' capacity to advance their research agendas.

\section{Limitation}

This study has some limitations that should be noted while interpreting the results. We did not include ASRH publications written in Portuguese and Arabic from SSA's Lusophone and Arab-speaking countries, potentially limiting the number of studies reviewed. Also, despite searching several databases and websites of relevant institutions, our search remains limited, given that ASRH studies may not be published online or in peer-reviewed journals.

\section{Conclusions}

In conclusion, our review demonstrated that research on ASRH is limited and unevenly distributed across SSA countries. Given the importance of research in advocacy and progressive ASRH policies, further investment in ASRH research and research capacity building is needed in the region. Future ASRH research should focus on under-researched countries, like Madagascar, Namibia, Togo, Liberia, Guinea Bissau, Equatorial Guinea, Mauritius, Mauritania, Chad or Eritrea. Prioritising ASRH research in these countries will in no small measure help to build the capacity of their local researchers. Research capacity building programmes should also prioritise these countries to strengthen local researchers' skills and enable them to chart their research priorities. Future studies should also focus on topics that have received limited attention, including early adolescence, gender norms, programme interventions, scaling up of interventions and policy evaluation.

Twitter Anthony Idowu Ajayi @aiajayi

Acknowledgements The authors are grateful to the research assistants who contributed to the data extraction and the entire Challenging the Politics of Social Exclusion team whose comments have helped improve this scoping review's focus.

Contributors AIA, EOO, BAU, MM and CWK made substantial contributions to this study's conceptualisation and implementation. AIA and EOO conducted the analysis and drafted the manuscript. All authors reviewed and revised the draft and approved the final version of the manuscript.

Funding This research was made possible through the funding to African Population and Health Research Center by the Swedish International Development Cooperation Agency for the Challenging the Politics of Social Exclusion project.

Map disclaimer The depiction of boundaries on this map does not imply the expression of any opinion whatsoever on the part of BMJ (or any member of its group) concerning the legal status of any country, territory, jurisdiction or area or of its authorities. This map is provided without any warranty of any kind, either express or implied.

Competing interests None declared.

Patient consent for publication Not required.

Ethics approval The study is exempt from ethical approval as it only uses publicly available publications.

Provenance and peer review Not commissioned; externally peer reviewed.

Data availability statement Data are available upon request from the corresponding author. 
Supplemental material This content has been supplied by the author(s). It has not been vetted by BMJ Publishing Group Limited (BMJ) and may not have been peer-reviewed. Any opinions or recommendations discussed are solely those of the author(s) and are not endorsed by BMJ. BMJ disclaims all liability and responsibility arising from any reliance placed on the content. Where the content includes any translated material, BMJ does not warrant the accuracy and reliability of the translations (including but not limited to local regulations, clinical guidelines, terminology, drug names and drug dosages), and is not responsible for any error and/or omissions arising from translation and adaptation or otherwise.

Open access This is an open access article distributed in accordance with the Creative Commons Attribution Non Commercial (CC BY-NC 4.0) license, which permits others to distribute, remix, adapt, build upon this work non-commercially, and license their derivative works on different terms, provided the original work is properly cited, appropriate credit is given, any changes made indicated, and the use is non-commercial. See: http://creativecommons.org/licenses/by-nc/4.0/.

\section{ORCID iDs}

Anthony Idowu Ajayi http://orcid.org/0000-0002-6004-3972

Emmanuel Oloche Otukpa http://orcid.org/0000-0002-9370-3500

\section{REFERENCES}

1 Bearak J, Popinchalk A, Alkema L, et al. Global, regional, and subregional trends in unintended pregnancy and its outcomes from 1990 to 2014: estimates from a Bayesian hierarchical model. Lancet Glob Health 2018;6:e380-9.

2 Morris JL, Rushwan H. Adolescent sexual and reproductive health: the global challenges. Int J Gynaecol Obstet 2015;131 Suppl 1:S40-2.

3 Woog V, Singh S, Browne A. Adolescent women's need for and use of sexual and reproductive health services in developing countries. New York: Guttmacher Institute, 2015: 1-63.

4 Neal S, Matthews Z, Frost M, et al. Childbearing in adolescents aged 12-15 years in low resource countries: a neglected issue. New estimates from demographic and household surveys in 42 countries. Acta Obstet Gynecol Scand 2012;91:1114-8.

5 Nove A, Matthews Z, Neal S, et al. Maternal mortality in adolescents compared with women of other ages: evidence from 144 countries. Lancet Glob Health 2014;2:e155-64.

6 Dellar RC, Dlamini S, Karim QA. Adolescent girls and young women: key populations for HIV epidemic control. J Int AIDS Soc 2015;18:19408.

7 World Health Organization. Global and regional estimates of violence against women: prevalence and health effects of intimate partner violence and non-partner sexual violence. Geneva, Switzerland: World Health Organization, 2013.

8 Shah IH, Åhman E. Unsafe abortion differentials in 2008 by age and developing country region: high burden among young women. Reprod Health Matters 2012;20:169-73.

9 Santhya KG, Jejeebhoy SJ. Sexual and reproductive health and rights of adolescent girls: evidence from low- and middle-income countries. Glob Public Health 2015;10:189-221.

10 Roth GA, Abate D, Abate KH, et al. Global, regional, and national age-sex-specific mortality for 282 causes of death in 195 countries and territories, 1980-2017: a systematic analysis for the global burden of disease study 2017. Lancet 2018;392:1736-88.

11 Sawyer SM, Afifi RA, Bearinger LH, et al. Adolescence: a foundation for future health. Lancet 2012;379:1630-40.

12 Johansen ER, Nielsen HS, Verner M. Long-Term consequences of early parenthood. J Marriage Fam 2020;82:1286-303.

13 Parsons J, Edmeades J, Kes A, et al. Economic impacts of child marriage: a review of the literature. Rev Faith Int Aff 2015;13:12-22.

14 Santelli JS, Sharma V, Viner R. 3. inequality, National wealth, economic development and global trends in teenage birth rates, 1990-2010. Journal of Adolescent Health 2013:52:S4-5.

15 Hobcraft J, Kiernan K. Childhood poverty, early motherhood and adult social exclusion. Br J Sociol 2001;52:495-517.

16 Lee $D$. The early socioeconomic effects of teenage childbearing: a propensity score matching approach. Demographic Research 2010;23:697-736

17 Birchall J. Early marriage, pregnancy and girl child school dropout. K4D Helpdesk report. Brighton: Institute of Development Studies, 2018.

18 Grønvik T, Fossgard Sandøy I, Sandøy IF. Complications associated with adolescent childbearing in sub-Saharan Africa: a systematic literature review and meta-analysis. PLoS One 2018;13:e0204327.
19 Chen X-K, Wen SW, Fleming N, et al. Increased risks of neonatal and postneonatal mortality associated with teenage pregnancy had different explanations. J Clin Epidemiol 2008;61:688-94.

20 Ganchimeg T, Ota E, Morisaki N, et al. Pregnancy and childbirth outcomes among adolescent mothers: a world Health organization multicountry study. BJOG 2014;121 Suppl 1:40-8.

21 Lachance CR, Burrus BB, Scott AR. Building an evidence base to inform interventions for pregnant and parenting adolescents: a call for rigorous evaluation. Am J Public Health 2012;102:1826-32.

22 Ajayi Al, Nwokocha EE, Adeniyi OV, et al. Unplanned pregnancyrisks and use of emergency contraception: a survey of two Nigerian universities. BMC Health Serv Res 2017;17:382.

23 Ajayi Al, Nwokocha EE, Akpan W, et al. Use of non-emergency contraceptive pills and concoctions as emergency contraception among Nigerian university students: results of a qualitative study. BMC Public Health 2016;16:1046.

24 Ajayi Al, Nwokocha EE, Akpan W. "It's Sweet Without Condom": Understanding Risky Sexual Behaviour Among Nigerian Female University Students. Online Journal of Health and Allied Sciences 2018;16.

25 Ajayi Al, Somefun OD. Transactional sex among Nigerian university students: the role of family structure and family support. PLoS One 2019;14:e0210349.

26 Chandra-Mouli V, McCarraher DR, Phillips SJ, et al. Contraception for adolescents in low and middle income countries: needs, barriers, and access. Reprod Health 2014;11:1.

27 Maina BW, Orindi BO, Sikweyiya Y, et al. Gender norms about romantic relationships and sexual experiences among very young male adolescents in Korogocho slum in Kenya. Int J Public Health 2020;65:497-506.

28 Moreau C, Li M, De Meyer S, et al. Measuring gender norms about relationships in early adolescence: results from the global early adolescent study. SSM Popul Health 2019;7:100314.

29 Commission AU. Agenda 2063: the Africa we want. Addis Ababa: African Union Commission, 2015.

30 Africa Union. State of African Population Report 2017. Keeping Rights of Girls, Adolescents and Young Women at the Centre of Africa's Demographic Dividend. Addis Ababa, Ethiopia, 2017.

31 Africa Union. AU roadmap on harnessing the demographic dividend through investments in youth. Addis Ababa, Ethiopia: African Union, 2017.

32 Africa Union. Maputo plan of action for implementing the continental policy framework on sexual and reproductive health and rights. Addis Ababa - Ethiopia: International Planned Parenthood Liaison Office to the African Union and ECA, 2014.

33 Africa Union Commission. Protocol to the African Charter on Human and People's Rights on the Rights of Women in Africa, 2003.

34 Africa Union Commission. Addis Ababa Declaration on population and development in Africa beyond 2014. Addis Ababa, Ethiopia: Development ARCoPa, 2013.

35 Viljoen F. An introduction to the protocol to the African charter on human and peoples' rights on the rights of women in Africa. Wash \& Lee J Civil Rts \& Soc Just 2009;16:11

36 Büyüm $\mathrm{AM}$, Kenney $\mathrm{C}$, Koris $\mathrm{A}$, et al. Decolonising global health: if not now, when? BMJ Glob Health 2020;5:e003394.

37 Abimbola S. The foreign gaze: authorship in academic global health, 2019.

38 Arksey H, O'Malley L. Scoping studies: towards a methodological framework. Int J Soc Res Methodol 2005;8:19-32.

39 Tricco AC, Lillie E, Zarin W, et al. PRISMA extension for scoping reviews (PRISMA-ScR): checklist and explanation. Ann Intern Med 2018;169:467-73.

40 Ajayi Al, Ushie BA, Mwoka M, et al. Mapping adolescent sexual and reproductive health research in sub-Saharan Africa: protocol for a scoping review. BMJ Open 2020;10:e035335.

41 Norton M, Chandra-Mouli V, Lane C. Interventions for preventing unintended, rapid repeat pregnancy among adolescents: a review of the evidence and lessons from high-quality evaluations. Glob Health Sci Pract 2017:5:547-70.

42 Chandra-Mouli V, Svanemyr J, Amin A, et al. Twenty years after International Conference on population and development: where are we with adolescent sexual and reproductive health and rights? J Adolesc Health 2015;56:S1-6.

43 Chandra-Mouli V, Lane C, Wong S. What does not work in adolescent sexual and reproductive health: a review of evidence on interventions commonly accepted as best practices. Global Health: Science and Practice 2015;3:333-40.

44 Chandra-Mouli V, Ferguson BJ, Plesons M, et al. The Political, Research, Programmatic, and Social Responses to Adolescent Sexual and Reproductive Health and Rights in the 25 Years Since 
the International Conference on Population and Development. $J$ Adolesc Health 2019;65:S16-40.

45 Kellermeyer L, Harnke B, Knight S. Covidence and Rayyan. JMLA 2018;106:580.

46 Hedt-Gauthier BL, Jeufack HM, Neufeld NH, et al. Stuck in the middle: a systematic review of authorship in collaborative health research in Africa, 2014-2016. BMJ Glob Health 2019;4:e001853.

47 Salam RA, Faqqah A, Sajjad N, et al. Improving adolescent sexual and reproductive health: a systematic review of potential interventions. J Adolesc Health 2016;59:S11-28.

48 Patton GC, Sawyer SM, Santelli JS, et al. Our future: a Lancet Commission on adolescent health and wellbeing. Lancet 2016;387:2423-78.

49 Kabiru CW, Izugbara CO, Beguy D. The health and wellbeing of young people in sub-Saharan Africa: an under-researched area? BMC Int Health Hum Rights 2013;13:11.

50 Izugbara CO, Kabiru CW, Amendah D. "It takes more than a fellowship program": reflections on capacity strengthening for health systems research in sub-Saharan Africa. Springer, 2017.

51 Fonn S, Ayiro LP, Cotton P, et al. Repositioning Africa in global knowledge production. Lancet 2018;392:1163-6.

52 Somefun OD, Adebayo KO. The role of mentoring in research ecosystems in sub-Saharan Africa: some experiences through the CARTA opportunity. Glob Public Health 2021;16:36-47.
53 Igumbor J, Bosire EN, Basera T. CARTA Fellows' Scientific Contribution to the African Public and Population Health Research Agenda (2011 to 2018, 2020.

54 Uwizeye D, Karimi F, Otukpa E, et al. Increasing Collaborative research output between early-career health researchers in Africa: lessons from the CARTA fellowship program. Glob Health Action 2020;13:1768795.

55 Chersich MF, Blaauw D, Dumbaugh M, et al. Local and foreign authorship of maternal health interventional research in low- and middle-income countries: systematic mapping of publications 20002012. Global Health 2016;12:35

56 Sawyerr A. African universities and the challenge of research capacity development. Journal of Higher Education in Africa/Revue de l'enseignement supérieur en Afrique 2004:213-42.

57 Chu KM, Jayaraman S, Kyamanywa $\mathrm{P}$, et al. Building research capacity in Africa: equity and global health collaborations. PLoS Med 2014;11:e1001612.

58 Simpkin V, Namubiru-Mwaura E, Clarke L, et al. Investing in health R\&D: where we are, what limits us, and how to make progress in Africa. BMJ Glob Health 2019;4:e001047.

59 Konde V. Towards achieving the African Union's recommendation of expenditure of $1 \%$ of GDP on research and development. ECA policy brief, No: ECA/18/004. United Nations Economic Commission for Africa, 2018.[Google Scholar] 2018. 